Wright State University

CORE Scholar

3-1-2007

\title{
Dominant Effect of Near-Interface Native Point Defects on ZnO Schottky Barriers
}

\author{
L. J. Brillson \\ H. L. Mosbacker \\ M. J. Hetzer \\ Y. Strzhemechny \\ David C. Look \\ Wright State University - Main Campus, david.look@wright.edu
}

See next page for additional authors

Follow this and additional works at: https://corescholar.libraries.wright.edu/physics

Part of the Physics Commons

\section{Repository Citation}

Brillson, L. J., Mosbacker, H. L., Hetzer, M. J., Strzhemechny, Y., Look, D. C., Cantwell, G., Zhang, J., \& Song, J. J. (2007). Dominant Effect of Near-Interface Native Point Defects on ZnO Schottky Barriers. Applied Physics Letters, 90 (10), 102116.

https://corescholar.libraries.wright.edu/physics/95

This Article is brought to you for free and open access by the Physics at CORE Scholar. It has been accepted for inclusion in Physics Faculty Publications by an authorized administrator of CORE Scholar. For more information, please contact library-corescholar@wright.edu. 


\section{Authors}

L. J. Brillson, H. L. Mosbacker, M. J. Hetzer, Y. Strzhemechny, David C. Look, G. Cantwell, J. Zhang, and J. J. Song

This article is available at CORE Scholar: https://corescholar.libraries.wright.edu/physics/95 


\title{
Dominant effect of near-interface native point defects on $\mathrm{ZnO}$ Schottky barriers
}

\author{
L. J. Brillson, ${ }^{\text {a) }}$ H. L. Mosbacker, ${ }^{\text {b) }}$ and M. J. Hetzer ${ }^{\text {b) }}$ \\ Department of Electrical and Computer Engineering, The Ohio State University, Columbus, Ohio 43210 \\ Y. Strzhemechny \\ Department of Physics and Astronomy, Texas Christian University, Fort Worth, Texas 76129
}

G. H. Jessen

Sensors Directorate, Air Force Research Laboratory, WPAFB, Ohio 45433

D. C. Look

Semiconductor Research Center, Wright State University, Dayton, Ohio 45433

and Materials and Manufacturing Directorate, Air Force Research Laboratory, WPAFB, Ohio 45433

G. Cantwell, J. Zhang, and J. J. Song ${ }^{\text {c) }}$

ZN Technology, Inc., 910 Columbia St., Brea, California 92821

(Received 10 January 2007; accepted 1 February 2007; published online 8 March 2007)

\begin{abstract}
The authors used depth-resolved cathodoluminescence spectroscopy and current-voltage measurements to probe metal- $\mathrm{ZnO}$ diodes as a function of native defect concentration, oxygen plasma processing, and metallization. The results show that resident native defects in $\mathrm{ZnO}$ single crystals and native defects created by the metallization process dominate metal-ZnO Schottky barrier heights and ideality factors. Results for $\mathrm{ZnO}(000 \overline{1})$ faces processed with room temperature remote oxygen plasmas to remove surface adsorbates and reduce subsurface native defects demonstrate the pivotal importance of crystal growth quality and metal-ZnO reactivity in forming near-interface states that control Schottky barrier properties. () 2007 American Institute of Physics. [DOI: $10.1063 / 1.2711536]$
\end{abstract}

As $\mathrm{ZnO}$ becomes an important candidate for next generation semiconductor electronics, ${ }^{1}$ it becomes critical to control its electrical contacts with metals. While different metals can produce a sizable range of Schottky barriers $(\mathrm{SBs}),{ }^{2}$ the wide variation of reported SBs for the same metal [e.g., Au (Refs. 1-5)] shows that stable and rectifying metal contacts to $\mathrm{ZnO}$ remain a challenge. Indeed, surface traps, adsorbates, ${ }^{3,4}$ processing, ${ }^{1,5}$ hydrogen, ${ }^{6}$ orientation, and surface dipoles ${ }^{7}$ can all affect $\mathrm{ZnO}$ 's surface and interface properties. Here we use nanoscale depth-resolved cathodoluminescence spectroscopy (DRCLS) coupled with surface science and electronic transport techniques to separate these extrinsic effects and show that near-interface native defects, both resident in the bulk and created by metallization, dominate rectification, recombination, and tunneling features of the metal- $\mathrm{ZnO}$ contact.

DRCLS measurement and remote plasma processing techniques used have been described previously. ${ }^{5,6,8}$ All spectra are taken at $10 \mathrm{~K}$ with constant power and shown without normalization. Room temperature, remote $\mathrm{O} / \mathrm{He}$ plasma (ROP) treatment yields clean, low energy diffractionordered $\mathrm{ZnO}$ polar surfaces with negligible added surface roughness. ${ }^{5}$ For incident electron beam energies $E_{B}=2,5,10$, and $20 \mathrm{keV}$, Monte Carlo simulations ${ }^{9}$ of the electron cascade yield depths of peak electron-hole pair creation rate of

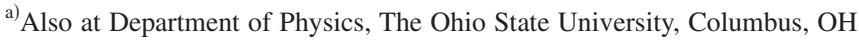
and Center for Materials Research, The Ohio State University, Columbus, $\mathrm{OH}$; electronic mail: brillson.1@ @osu.edu

b) Also at Department of Physics, The Ohio State University, Columbus, $\mathrm{OH}$.

c) Also at Department of Electrical and Computer Engineering, University of California, San Diego, CA.
}

$U_{0} \sim 55,85,330$, and $990 \mathrm{~nm}$, respectively, below the bare $\mathrm{ZnO}$ surface. Peak excitation through 30-nm-thick metal diodes for $E_{B}=2,5,10$, and $20 \mathrm{keV}$ corresponds to $U_{0} \sim-20$ (inside the metal), 40, 270, and $960 \mathrm{~nm}$, respectively, varying slightly with metal density. For most metals, a $5 \mathrm{keV}$ beam through the $30-\mathrm{nm}$-thick metal overlayer and a $2 \mathrm{keV}$ beam into the free $\mathrm{ZnO}$ surface have comparable $U_{0}$. Metal diodes (sets of five to six each, $0.4 \mathrm{~mm}$ diameter) were patterned on as-received (AR) or ROP-treated surfaces either in ultrahigh vacuum (UHV) by filament evaporation without any intervening air exposure or by electron beam evaporation following air exposure. Schottky barriers and ideality factors were extracted from current-voltage $(I-V)$ curves using thermionic field emission analysis. UHV Au deposition on similar ROP-cleaned, low-defect $\mathrm{ZnO}$ samples produced nearly identical $I-V$ characteristics, indicating that adsorbates on ROP-cleaned surfaces have only secondary effects. For $\mathrm{Au}-\mathrm{ZnO}$ contacts, $\mathrm{O}$ and $\mathrm{Zn}$ polar orientations showed similar $I-V$ behavior. ${ }^{5}$

We examined $\mathrm{ZnO}$ single crystals grown hydrothermally, by chemical vapor transport, and by pressurized melt, finding near band edge (NBE) and deep level defect emissions at $\sim 2.1,2.5$, and $3 \mathrm{eV}$ whose absolute and relative intensities varied by orders of magnitude. ${ }^{10}$ Depending on whether defect intensities, e.g., $I(2.5 \mathrm{eV})$, are comparable to or orders of magnitude below $I(\mathrm{NBE})$, these crystals are termed "high defect" or "low defect," respectively. We used ROP treatments not only to clean the AR surfaces but also to remove $\mathrm{H}$ and near-surface defects. Figure 1(a) shows the relative defect intensity $I(2.5 \mathrm{eV}) / I(\mathrm{NBE})$ changes both versus depth and with increasing ROP exposure for a high-defect $\mathrm{ZnO}$ 

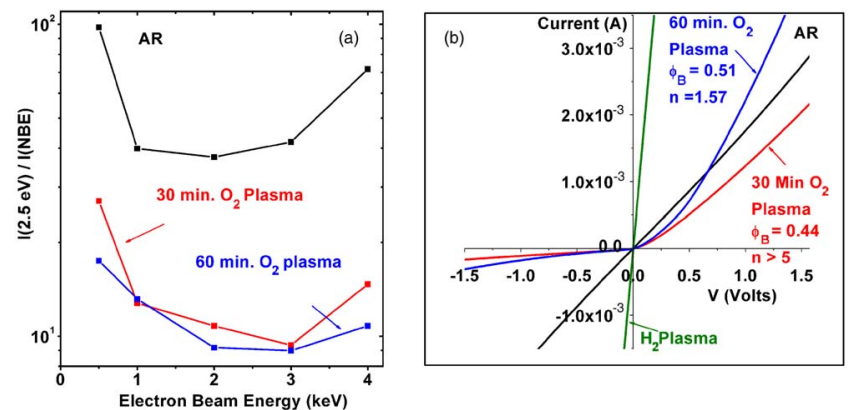

FIG. 1. (Color online) (a) Relative defect intensity changes vs depth and vs ROP exposure. (b) Corresponding $I-V$ plots plus effect of $\mathrm{H}_{2}$ plasma exposure for Au diodes on these surfaces.

crystal. For $E_{B}=1-4 \mathrm{keV}$, the $2.5 \mathrm{eV}$ defect intensity increases with proximity to the surface. ${ }^{5}$ Within the top $\sim 100 \mathrm{~nm}$, these defects decrease with increasing ROP exposure time, even after obtaining a clean surface $(30 \mathrm{~min})$. A 60 min treatment decreases $I(2.5 \mathrm{eV}) / I(\mathrm{NBE})$ by nearly an order of magnitude within nanometers of the surface. The $I-V$ characteristics for Au diodes on the same surface corresponding to the treatments in Fig. 1(a) show monotonic changes from Ohmic to Schottky-like behavior, an increase in barrier height $\Phi_{B}$ (corrected for image force lowering), and a decrease in ideality factor with increasing ROP exposure. Figure 1(b) also shows that $\mathrm{H}_{2}$ plasma treatment of the 60 min $\mathrm{O}_{2}$-treated surface converts such barriers back to an Ohmic contact with even lower contact resistanceconsistent with hydrogen's donor character. ${ }^{6,11}$ Thus ROP cleaning can be used to minimize variations in $\mathrm{ZnO}$ surfaces due to surface adsorbates, subsurface defects, and hydrogen.

Figure 2 demonstrates that subsurface native defects have a major impact on Schottky barrier properties. For 60 min ROP-cleaned $\mathrm{ZnO}(000 \overline{1})$ surfaces, Fig. 2(a) exhibits nearly three order-of-magnitude variation between lowdefect (ZN Tech) and high-defect crystals. For Au diodes deposited on these surfaces, Fig. 2(b) shows $I-V$ curves ranging from Ohmic (high defect) to rectifying (medium defect, $\Phi_{B}=0.43 \mathrm{eV}, n=3.5$ ), to strongly rectifying (low defect, $\Phi_{B}=0.48 \mathrm{eV}, n=1.30$ ) with reverse currents $I_{R}$ decreasing by over two orders of magnitude as defect densities decrease. $\mathrm{X}$-ray photoemission spectroscopy measurements of Fermi level movement with plasma cleaning show similar rigid core level shifts of $>0.75 \mathrm{eV} n$-type band bending in each case. The $I_{R}$ increase with increasing defect density is consistent with either impurity band transport or increased doping and tunneling through a narrowed depletion region. Such
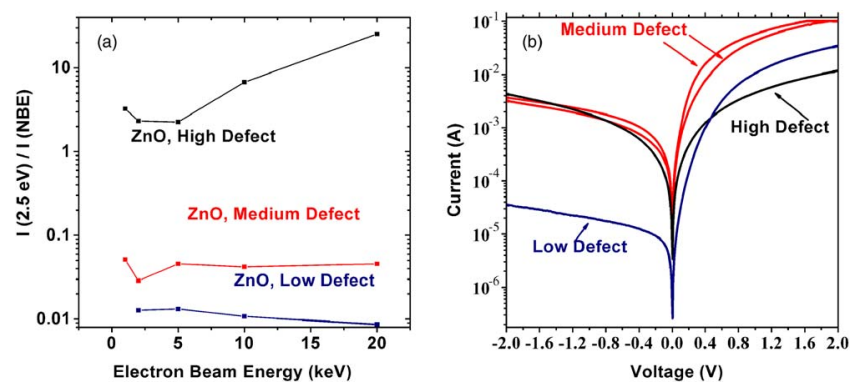

FIG. 2. (Color online) (a) $I(2.5 \mathrm{eV}) / I(\mathrm{NBE})$ vs $E_{B}$ and (b) corresponding $I-V$ curves showing order of magnitude increases in defect emission and reverse current for low-, medium-, and high-defect, ROP-cleaned $\mathrm{ZnO}(000 \overline{1})$.
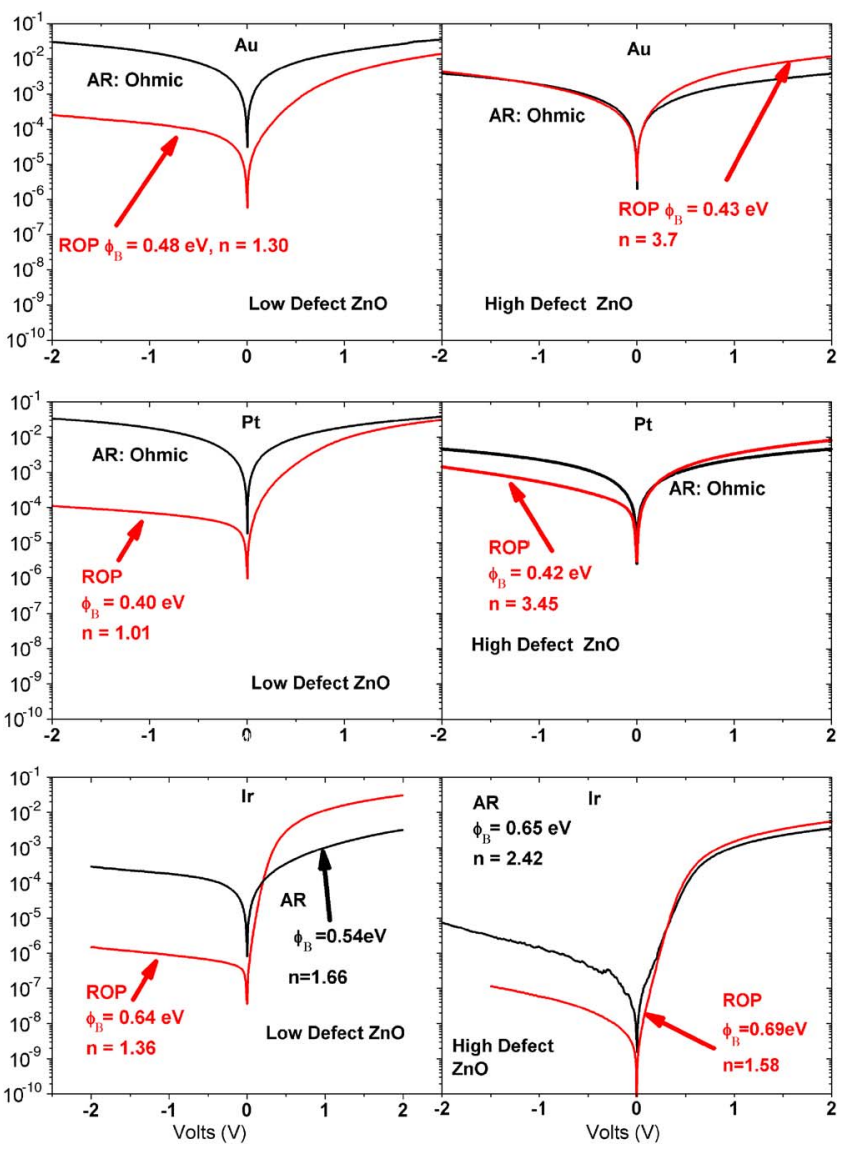

FIG. 3. (Color online) $I-V$ curves for Au, Pt, and Ir on the same low-defect (left) vs high-defect (right) $\mathrm{ZnO}(000 \overline{1})$ surface showing higher barriers and lower ideality factors for ROP-treated vs AR surfaces. Low-defect diodes all show lower ideality factors.

carrier concentration increases are evident from capacitancevoltage $(C-V)$ measurements of Ir diodes on ROP-cleaned, low-defect $\mathrm{ZnO}(000 \overline{1})$ showing an increase from 3.5 $\times 10^{16}$ to $>10^{17} \mathrm{~cm}^{-3}$ from a depth of $\sim 200-\sim 90 \mathrm{~nm}$ from the surface. ${ }^{12}$ Deep level CL emissions show a corresponding $3 \times$ increase in $I(2.5 \mathrm{eV})$ for $U_{0} \sim 75$ vs $220 \mathrm{~nm}$. Carrier concentrations extrapolated to the surface would produce depletion widths of only a few tens of nanometers, thereby increasing tunneling. Deep level optical spectroscopy show multiple deep levels across the band gap, including a level $2.7 \mathrm{eV}$ above ( $0.6 \mathrm{eV}$ below) the valence band maximum $E_{V}$ (conduction band minimum $E_{C}$ ) at room temperature. Similarly, deep level transient spectroscopy reveals a donor level with a concentration of $3.35 \times 10^{14} \mathrm{~cm}^{-3}$ at $E_{C}-0.55 \mathrm{eV}$. Thus deep levels and doping are changing within the $\mathrm{ZnO}$ depletion region.

Figure 3 shows the effect of ROP cleaning and deep level concentration on $I-V$ SBs for Au, Pt, and Ir diodes. For both $\mathrm{Au}$ and Pt on high- or low-defect AR $\mathrm{ZnO}$, initial contacts are Ohmic, becoming rectifying with ROP cleaning and defect reduction. For Ir, the initially rectifying contacts become more so with ROP cleaning. For each metal, (a) ROP treatment produces higher barriers and lower ideality factors and (b) low-defect $\mathrm{ZnO}$ yields lower (i.e., closer to unity) ideality factors.

Metal deposition also produces native defects at $\mathrm{ZnO}$ surfaces. Figure 4 shows DRCLS spectra taken with $E_{B}=2$ or $5 \mathrm{keV}$ for the bare or $30 \mathrm{~nm}$ metal-covered $(000 \overline{1})$ surface, 

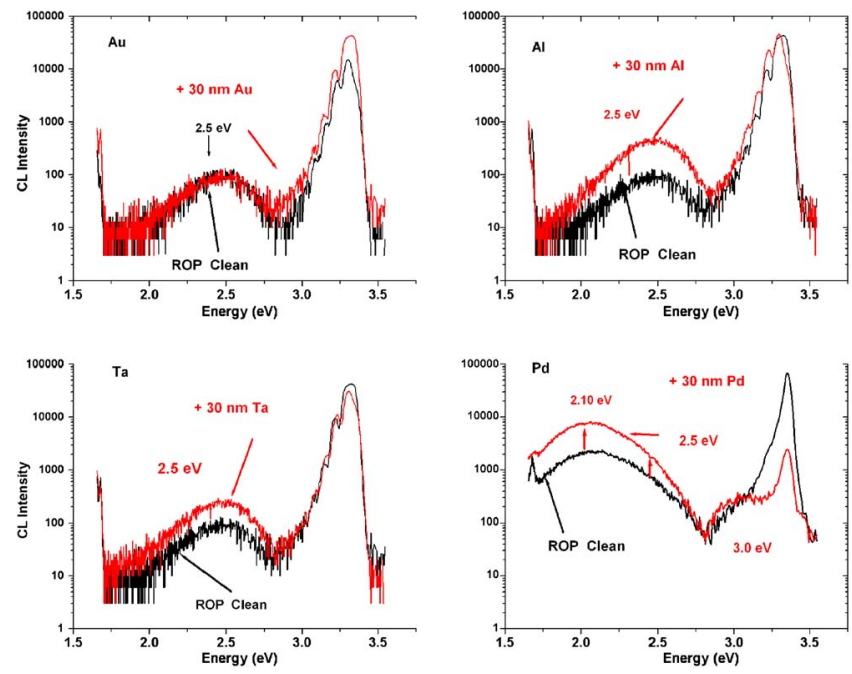

FIG. 4. (Color online) Room temperature deposition of $30 \mathrm{~nm} \mathrm{Au}, \mathrm{Al}$, and Ta on low- (Pd on medium-) defect ROP-cleaned $\mathrm{ZnO}(000 \overline{1})$ showing $\mathrm{Al}$, Ta, and $\mathrm{Pd}$ induced increases in near-surface defect emission.

respectively. Besides a broadened NBE peak, roomtemperature-deposited $\mathrm{Au}$ overlayers induce no deep level features, whereas $\mathrm{Al}$, Ta, and $\mathrm{Pd}$ induce emission that appears at $2.5 \mathrm{eV}$. For $\mathrm{Pd}$, emission appears at $2.1 \mathrm{eV}$ that dominates. Relative to the bare surface, these emissions are localized within tens of nanometers of the interface. Metalinduced deep levels are also found for high-defect $\mathrm{ZnO}$ but are less clearly distinguished from defects already resident in the crystal. These and other metals (i.e., Pt and Ir) produce defect changes only at 2.1, 2.5, or $3 \mathrm{eV}$. Deep level emissions at these energies are characteristic of $\mathrm{ZnO}$ crystals in general and change with O- vs Zn-rich growth conditions. ${ }^{13}$ Theory shows that $\mathrm{Zn}$ and $\mathrm{O}$ vacancies are the most stable defects in $\mathrm{ZnO} .{ }^{14}$ Defects near $E_{C}-2.1 \mathrm{eV}$ can be assigned to $\mathrm{Zn}$ vacancies with positron annihilation spectroscopy. ${ }^{15} \mathrm{Op}-$ tically detected paramagnetic resonance measurements ${ }^{16}$ and theory ${ }^{17}$ suggest that $\mathrm{O}$ vacancy levels lie at $E_{V}+2.48 \mathrm{eV}$. Thus, metals induce deep level emissions characteristic of native point defects common with the semiconductor bulk rather than states unique to surface adsorption. ${ }^{18}$ Indeed such defects extend beyond the surface into the bulk. Analogous features are induced for metals on $\mathrm{SiC} .{ }^{19}$ Native point defects at and below the $\mathrm{ZnO}$ surface are present that dominate contact rectification and ideality. Their deep level densities vary by orders of magnitude at and below the surface, depending on growth, surface processing, and metallization, and must be minimized to control $\mathrm{ZnO}$ contacts.

The authors gratefully acknowledge support for this work by National Science Foundation Grant No. DMR0513968 (Verne Hess), AFRL Contract No. FA8650-06D5401 (J. Zetts), and Michael Callahan (Hanscom Air Force Base) for representative $\mathrm{ZnO}$ crystals from vendors worldwide.

${ }^{1}$ S. J. Pearton, D. P. Norton, K. Ip, Y. W. Heo, and T. Steiner, Prog. Mater. Sci. 50, 312 (2005), and references therein.

${ }^{2}$ C. A. Mead, Solid-State Electron. 9, 1023 (1966).

${ }^{3}$ H. Moorman, D. Kohl, and G. Heiland, Surf. Sci. 100, 302 (1980), and references therein.

${ }^{4}$ B. J. Coppa, R. F. Davis, and R. J. Nemanich, Appl. Phys. Lett. 82, 400 (2003).

${ }^{5}$ H. L. Mosbacker, Y. M. Strzhemechny, B. D. White, P. E. Smith, D. C. Look, D. C. Reynolds, C. W. Litton, and L. J. Brillson, Appl. Phys. Lett. 87, 012102 (2005), and references therein.

${ }^{6}$ Y. M. Strzhemechny, H. L. Mosbacker, D. C. Look, D. C. Reynolds, C. W. Litton, N. Y. Garces, N. C. Giles, L. E. Halliburton, S. Niki, and L. J. Brillson, Appl. Phys. Lett. 84, 2545 (2004).

${ }^{7}$ H. Sheng, S. Muthukamar, N. W. Emanetoglu, and Y. Lu, Appl. Phys. Lett. 80, 2132 (2002).

${ }^{8}$ L. J. Brillson, J. Vac. Sci. Technol. B 19, 1762 (2001).

${ }^{9}$ D. Drouin, P. Hovington, and R. Gauvin, Scanning 19, 20 (1997).

${ }^{10}$ H. L. Mosbacker, D. C. Look, and L. J. Brillson (unpublished).

${ }^{11}$ C. G. Van de Walle, Phys. Rev. Lett. 85, 1012 (2000); Physica B 308, 899 (2001); Phys. Status Solidi B 229, 221 (2002).

${ }^{12}$ H. L. Mosbacker, S. El Hage, M. Gonzalez, S. A. Ringel, M. Hetzer, D. C. Look, G. Cantwell, J. Zhang, J. J. Song, and L. J. Brillson, J. Vac. Sci. Technol. B (in press).

${ }^{13}$ H. C. Ong and G. T. Du, J. Cryst. Growth 265, 471 (2004).

${ }^{14}$ A. F. Kohan, G. Ceder, D. Morgan, and C. G. Van de Walle, Phys. Rev. B 61, 15019 (2000).

${ }^{15}$ F. Tuomisto, K. Saarinen, D. C. Look, and G. C. Farlow, Phys. Rev. B 72, 085206 (2005).

${ }^{16}$ L. S. Vlasenko and G. D. Watkins, Phys. Rev. B 71, 125210 (2005).

${ }^{17}$ A. Janotti and C. G. Van de Walle, Appl. Phys. Lett. 87, 122102 (2005).

${ }^{18}$ W. E. Spicer, I. Lindau, P. Skeath, C. Y. Su, and P. Chye, Phys. Rev. Lett. 40, 420 (1980).

${ }^{19}$ S. Tumakha, S. H. Goss, L. J. Brillson, and R. S. Okojie, J. Vac. Sci. Technol. B 23, 594 (2005). 